\title{
Avaliação da Capacidade Funcional e da Limitação Física em Sujeitos Pós-Revascularização Miocárdica
} Assessment of Functional Capacity and Physical Limitation in Subjects Submitted to Myocardial Revascularization

\author{
CAIO CESAR ARAÚJO MORAIS 1 \\ TAINÁ MARIA DE SOUZA VIDAL ${ }^{1}$ \\ GILMÁRIO RICARTE BATISTA ${ }^{2}$ \\ EDUARDO ÉRIKO TENÓRIO DE FRANÇA ${ }^{3}$ \\ JADER CARNEIRO JÚNIOR (IN MEMORIAN) ${ }^{3}$
}

\section{RESUMO}

Objetivo: Avaliar a capacidade funcional e a limitação física, em pacientes submetidos à cirurgia de revascularização do miocárdio (CRVM) com seis meses do pós-operatório. Material e Métodos: Foram estudados 28 pacientes com doença arterial coronariana, submetidos à CRVM entre novembro de 2008 e abril de 2009. Foram avaliadas a capacidade funcional e a limitação por aspectos físicos pela apuração dos respectivos domínios presentes no questionário padronizado Medical Outcomes Study - short form 36 (MOS-SF), e o nível de atividade física, por meio do International Physical Activity Questionnaire (IPAQ). Resultados: Houve diferenças significantes entre o pré e 0 pós-operatório para os domínios "capacidade funcional" ( 41,79 vs. 70,$18 ; p<0,001)$ e "limitação por aspectos físicos" ( 3,57 vs. 35,$71 ; p<0,001)$. Foi evidenciado que os pacientes classificados como "ativos" pelo IPAQ tiveram pontuação significativamente maior do que o classificado com "sedentário" para o domínio "capacidade funcional" $(81,07$ vs. 45,$00 ; p=0,012$ ) e que os classificados como "insuficientemente ativo" (58,93 vs. 11,$11 ; p=0,006)$ e "sedentário" (58,93 vs. 15,$00 ; p=0,006)$ no domínio "limitação por aspectos físicos". Conclusão: Foi observado acréscimo na pontuação dos domínios "capacidade funcional" e "limitação por aspectos físicos" após seis meses da CRVM, o que traduz em aumento da capacidade de realizar atividades que no pré-operatório não eram possíveis. A melhora destes domínios foi mais expressiva em indivíduos que praticaram atividade física regularmente após o procedimento cirúrgico.

\section{DESCRITORES}

Atividade Motora. Infarto do Miocárdio. Estilo de Vida Sedentário.

\begin{abstract}
Objective: To evaluate the functional capacity and physical limitation in patients submitted to coronary artery bypass graft (CABG) at six months after surgery. Material and Methods: 28 patients with coronary artery disease who underwent CABG between November 2008 and April 2009 were included in this study. Their functional capacity and physical limitation were approached considering the respective domains present in the standardized questionnaire Medical Outcomes Study Short Form (MOS-SF), as well as the level of physical activity through the International Physical Activity Questionnaire (IPAQ), in the period of six months after the CABG. Results: There were significant differences before and after surgery for the domains "functional capacity" (41.79 vs. $70.18 ; p$ $<0.001$ ) and "limited by physical aspects" (3.57 vs. $35.71 p$ $<0.001)$. It was shown that patients classified as "active" by IPAQ had significantly higher scores than those rated as "sedentary" for the domain "functional capacity" (81.07 vs. $45.00 ; p=0.012$ ) and also higher than those classified as "insufficiently active" (58.93 vs. $11.11 ; p=0.006)$ and "sedentary" (58.93 vs. $15.00 ; p=0.006)$ for the domain "limited by physical aspects." Conclusion: There was an increase in the scores of the domains "functional capacity" and "limitation by physical aspects" six months after CABG, which translates into increased ability to perform activities that could not be performed preoperatively. The improvement of these areas was more significant in subjects who practiced regular physical activity after surgery.
\end{abstract}

\section{DESCRIPTORS}

Motor Activity. Myocardial Infarction. Sedentary Lifestyle.

\footnotetext{
1 Fisioterapeuta, especialista em Fisioterapia Cardiorrespiratória pela Universidade Federal de Pernambuco, Recife, PE - Brasil.

2 Professor Adjunto do Departamento de Educação Física da Universidade Federal de Pernambuco (UFPE), Recife, PE - Brasil.

3 Professor Adjunto do Departamento de Fisioterapia da Universidade Católica de Pernambuco (UNICAP), Recife, PE - Brasil.
} 
A s enfermidades cardiovasculares são as causas mais frequentes de morbimortalidade no Brasil, apresentando aumento em todas as faixas etárias no estado de Pernambuco no período entre 1980 e $1998^{1}$, com predomínio do infarto agudo do miocárdio (IAM) e da insuficiência cardíaca congestiva (ICC) como complicações da doença arterial coronariana (DAC) ${ }^{2}$. Objetivando minimizar as alterações funcionais do coração $^{3}$, diminuir a mortalidade ${ }^{4}$, a limitação física e os sintomas da angina ${ }^{5}$, tem-se indicado a cirurgia de revascularização do miocárdio (CRVM) como uma das opções no tratamento destes indivíduos.

Em pacientes submetidos à CRVM, os programas de reabilitação cardiopulmonar e metabólica (RCPM), incluindo as diversas fases existentes, reduzem ainda mais as taxas de morbidade e mortalidade ${ }^{6}$, visando a melhoria no condicionamento físico, proporcionando o retorno às atividades profissionais e esportivas ${ }^{7}$. Todavia, acredita-se que boa parte dos pacientes aptos ao programa de RCPM supervisionada não tenha acesso ao mesmo, por dificuldades de ordem econômica, social e cultural, dessa forma, tornando-se subutilizada ${ }^{8,9}$.

Estudos mostram que, a prática de exercício físico, promove diminuição na incidência de fibrilação atrial $^{10}$, aumenta a reserva de perfusão miocárdica ${ }^{11} \mathrm{e}$ melhora diversos outros parâmetros fisiológicos ${ }^{12,13}$, muito embora, acredita-se que um número reduzido desses estudos tem avaliado se essas mudanças na aptidão física afetam a percepção do paciente quanto ao seu estado funcional ${ }^{14,15}$.

Desse modo, o presente estudo teve como objetivo avaliar a capacidade funcional e a limitação física, em pacientes submetidos à CRVM com seis meses do pós-operatório.

\section{MATERIAL E MÉTODOS}

Realizou-se um estudo observacional do tipo coorte e prospectivo, desenvolvido entre novembro de 2008 e abril de 2009, no Real Hospital Português de Beneficência em Pernambuco (RHP).

Foram recrutados 49 pacientes, com idade entre 41 e 75 anos, admitidos com diagnóstico de isquemia miocárdica clínica e funcional pelo Instituto do Coração de Pernambuco. Todos os pacientes aguardaram em uma lista de espera para a CRVM, sob recomendação de repouso quanto à realização de atividades do trabalho, do lar ou exercício físico. Foram excluídos pacientes com deficiência física, doença neurológica, ortopédica e alterações psíquicas que impossibilitassem a avaliação e/ou a prática de atividade física após a cirurgia.
Os fatores de risco para doenças cardiovasculares existentes como hipertensão arterial sistêmica (HAS), sedentarismo, histórico familiar, diabetes mellitus, tabagista, dislipidemias, aterosclerose, Doença Pulmonar Obstrutiva Crônica (DPOC) e o Índice de Massa Corporal (IMC), foram recolhidos por meio de prontuários padronizados no momento da admissão hospitalar.

A avaliação da capacidade funcional e da limitação por aspectos físicos foi realizada pelos domínios do questionário Medical Outcomes Study short form (MOS SF-36) ${ }^{16}$. Os itens são classificados em uma escala de 0 a 100, de modo que essas pontuações indicam a pior e a melhor percepção da saúde, respectivamente. Os dois domínios foram avaliados em dois momentos distintos, estando a primeira entrevista no pré-cirúrgico, enquanto que o segundo momento se deu por meio de uma entrevista telefônica depois de completados seis meses da CRVM.

O nível de atividade física foi classificado pelo International Physical Activity Questionnaire (IPAQ), em sua versão curta ${ }^{18,19}$, composto de seis perguntas relacionadas ao tempo que o paciente gastou realizando atividade física na última semana. De acordo com as respostas cedidas, o sujeito pode ser classificado como: sedentário, insuficientemente ativo, ativo e muito ativo. O IPAQ foi aplicado após seis meses da cirurgia, juntamente com os dois domínios.

Os questionários utilizados são validados na língua portuguêsa ${ }^{16}$ e possuem aceitabilidade e confiabilidade comprovadas ${ }^{16,18,19}$.

O presente estudo foi aprovado pelo Comitê de Ética de Ensino e Pesquisa da Fundação de Hematologia e Hemoterapia de Pernambuco - HEMOPE (043/2008), conforme as normas envolvendo pesquisas com seres humanos na Resolução 196/96 do Conselho Nacional de Saúde. Todos os pacientes receberam as informações decorrentes de suas avaliações e assinaram, previamente, o Termo de Consentimento Livre e Esclarecido.

Para análise dos dados foi utilizado o programa Statistical Package for Social Sciences (SPSS - 17.0), por meio de estatística descritiva (distribuição de frequência, média, desvio padrão, mínimo e máximo) e inferencial (teste t pareado para amostras dependentes e Análise de variância - ANOVA), com nível de significância pré-estabelecido de $5 \%$. O teste t pareado foi utilizado para verificar diferenças entre o pré e após 6 meses da RCVM e a ANOVA para comparar a capacidade funcional e da limitação física de acordo com o nível de atividade física. 


\section{RESULTADOS}

De 49 pacientes recrutados, 28 fizeram parte da amostra final (Figura 1), com idade média de 58,33 $\pm 13,80$ anos, sendo este grupo composto por $15(53,5 \%)$ do gênero masculino e $13(46,4 \%)$ do feminino.

As características dos pacientes que concluíram o estudo quanto ao IMC e fatores de risco para DAC estão distribuídas na Tabela 1. Nesta, verifica-se que os fatores de risco mais prevalentes foram: a hipertensão arterial sistêmica-HAS (82,1\%), o sedentarismo (50,0\%), histórico familiar (46,4\%), e Diabetes Mellitus (42,8\%). Além disso, quanto ao IMC 21,4\% da amostra estudada foram classificados como eutrófico, $60,7 \%$ como sobrepeso e $17,8 \%$ como obesidade grau I.

Em relação aos domínios estudados, houveram diferenças significantes entre o pré e após seis meses da cirurgia $(\mathrm{P}<0,001)$, conforme é mostrado na Tabela 2. Na capacidade funcional, os pacientes apresentaram uma evolução de 28,39 pontos, e na limitação por aspectos físicos um aumento de 32,14 pontos.

Quanto ao nível de atividade física após 6 meses da CRVM, 50,0\% da amostra foram classificadas como "ativos", seguidos de 32,1\% como "insuficientemente ativos" e, 17,9\% como "sedentários". Nenhum dos pacientes apresentou nível classificado como "muito ativo".

Na comparação da capacidade funcional e da limitação por aspectos físicos, de acordo com o nível de atividade física após o sexto mês, evidenciou-se que os pacientes classificados como ativo apresentaram pontuação significativa maior na capacidade funcional

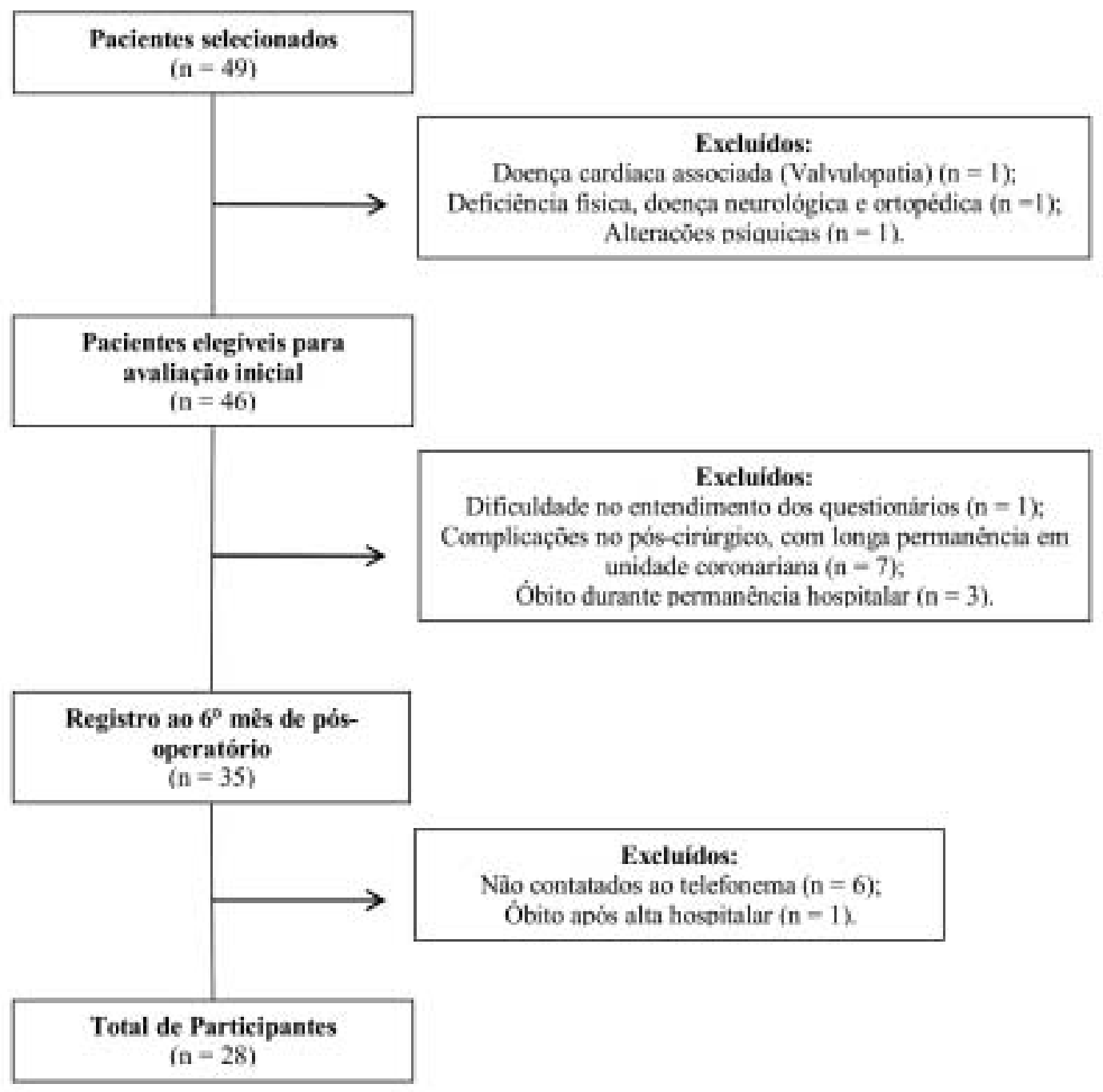

Fiqura 1. Fluxoarama do estudo quanto aos critérios de exclusão ao lonqo de seis meses, 


\begin{tabular}{|c|c|c|}
\hline & & \\
\hline \multicolumn{3}{|l|}{ Fatores de Risco } \\
\hline Hipertensão Arterial Sistêmica & 23 & 82,1 \\
\hline Sedentarismo & 14 & 50.0 \\
\hline Histórico familiar & 13 & 46,4 \\
\hline Diabetes Mellitus & 12 & 42,8 \\
\hline Tabacista & 7 & 25.0 \\
\hline Dislipidemias & 5 & 17.8 \\
\hline Aterosclerose & 3 & 10.7 \\
\hline \multicolumn{3}{|l|}{ Doença Pulmonar Obstrutiva } \\
\hline Crònica & 1 & 3.5 \\
\hline \multicolumn{3}{|l|}{ Indice de Massa Corporal } \\
\hline Eutrófico & 6 & 21,4 \\
\hline Sobrepeso & 17 & 60,7 \\
\hline Obesidade arau I & 5 & 17.8 \\
\hline
\end{tabular}

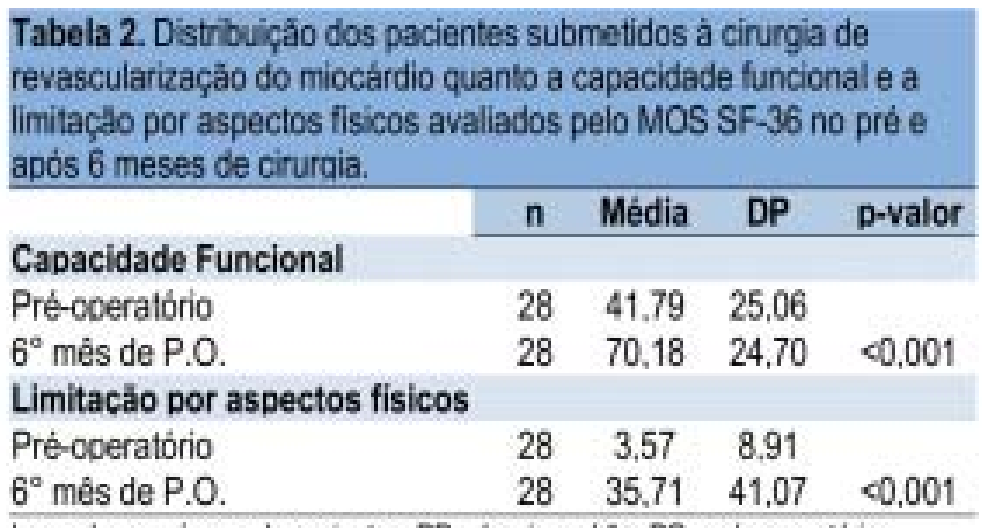

Legenda: $n=$ número de pacientes, DP= desvio padrâo, $P O=$ = pos-ogeratario.

apenas quando comparados com os pacientes sedentários $(P=0,012)$. Ao analisar a limitação por aspectos físicos, os pacientes classificados como ativo apresentaram escores significativos melhores que os outros dois. Os pacientes classificados como insuficientemente ativo e sedentário apresentaram pontuação muito baixa, 11,11 e 15,00, respectivamente, no domínio supracitado, conforme a Tabela 3.

\section{DISCUSSÃO}

As análises dos fatores de risco e do IMC em nossa pesquisa mostram que os preditores para a DAC e posterior indicação cirúrgica foram a HAS, o sedentarismo, o histórico familiar, a Diabetes Mellitus e o sobrepeso, fatores estes constatados em estudo prévio conduzido em 20 centros médicos no Brasil ${ }^{20}$.

Somente os domínios capacidade funcional e limitação física foram analisados, tendo em vista que os demais existentes no questionário MOS SF-36, sofrem influência negativa de fatores emocionais, a exemplo da depressão ${ }^{21}$, portanto, sendo o nível de atividade física fator determinante para correlação com os dois domínios anteriormente citados.

De acordo com a escala que varia entre 0 e 100 , onde zero é o pior escore e cem é o melhor, percebe-se que a capacidade funcional desses pacientes encontra- 


\begin{tabular}{|c|c|c|c|c|c|c|c|}
\hline Capacidade Funcional & $\mathbf{n}$ & Minimo & Máximo & Média & DP & \multicolumn{2}{|c|}{ D-valor } \\
\hline Sedentário & 5 & 5.00 & 75,00 & 45,00 & 34.82 & & \\
\hline Insuficientemente ativo & 9 & 25,00 & 95,00 & 67,22 & 22.65 & 0,012 & $\mathrm{~A} \times \mathrm{S}$ \\
\hline Ativo & 14 & 50,00 & 100,00 & 81,07 & 14,17 & & \\
\hline \multicolumn{8}{|l|}{$\begin{array}{l}\text { Limitação por aspectos } \\
\text { físicos }\end{array}$} \\
\hline Sedentário & 5 & 0,00 & 50,00 & 15,00 & 22,36 & & $A \times S$ \\
\hline Insuficientemente ativo & 9 & 0.00 & 50,00 & 11.11 & 22.05 & 0,006 & $A x$ \\
\hline Ativo & 14 & 0,00 & 100,00 & 58,93 & 43.42 & & IA \\
\hline
\end{tabular}

Legenda: $\mathrm{S}=$ sedentário, $\mathrm{A}=$ afivo, $\mid \mathrm{A}=$ insuficientemente atwo, $\mathrm{DP}=$ desvio padréco

se, em longo prazo, próxima da melhor percepção de saúde.

Já a limitação por aspectos físicos, ainda permaneceu em baixos índices, o que significa a baixa capacidade de realizar as atividades diárias.

A CRVM é descrita como a intervenção que apresentou melhores resultados no questionário MOS SF-36, quando comparada a angioplastia, e o tratamento medicamentoso ${ }^{25}$. No grupo dos pacientes submetidos a revascularização, 83,5\% melhoraram no domínio "capacidade funcional" e 65,5\% no domínio "limitação física". Contudo, no estudo não foi apontado se os pacientes realizavam algum tipo de atividade física.

Existe evidência da melhora na função física após 6 meses de revascularização do miocárdio em praticantes ou não de exercício físico supervisionado ${ }^{14}$, entretanto, o ganho no grupo de praticantes foi significantemente maior $(\mathrm{p}<0,001)$. Observou-se ainda que apesar de promover a manutenção do estilo de vida saudável, a adesão à reabilitação cárdica permanecia baixa, com menos de 1/4 das pessoas elegíveis participando.

No presente estudo foi verificado que $50,0 \%$ dos pacientes encontravam-se com o nível de atividade física classificado como ativo e nenhum como muito ativo. Acredita-se que a orientação clínica no momento da alta, quanto à realização de atividade física, bem como o prosseguimento aos exercícios praticados durante o internamento, não foi seguida corretamente pelos pacientes classificados como sedentário $(17,9 \%)$ e insuficientemente ativo $(32,1 \%)$.

Um estudo com 2.298 pacientes de 19 centros dos Estados Unidos ${ }^{26}$ avaliou se os pacientes após IAM recordavam e cumpriam 13 recomendações para a prevenção secundária, como a prática de exercício, o uso correto das medicações, a modificação da dieta e a cessação tabágica. Foi encontrado que 19,0\% respeitavam todas as instruções, e 33,0\% do grupo praticavam exercício físico supervisionado. Os autores constataram associação entre a aderência às instruções e as características sociais da amostra, estando os indivíduos mais jovens e de baixa condição social com menor adesão.

Quanto à comparação realizada no domínio capacidade funcional entre os pacientes classificados de acordo com o nível de atividade física, acredita-se que os pacientes considerados ativos apresentam pouca dificuldade para realizar atividades moderadas, devido à alta pontuação encontrada $(81,07)$. Pode-se considerar o inverso para os pacientes classificados como sedentário, pelo fato de apresentarem valores significativamente menores que os pacientes designados ativos.

No entanto, ainda deve existir certa dificuldade no grupo ativo para fazer atividades rigorosas, que exigem mais esforço, já que nenhum paciente relatou ter praticado atividades mais vigorosas, como correr, após 6 meses de pós-operatório.

Ao analisar as comparações na limitação por aspectos físicos, os pacientes classificados como ativos obtiveram escores expressivamente melhores que os insuficientemente ativos e sedentários. Vale salientar os escores obtidos pelos sedentários e insuficientemente ativos, que atingiram níveis muito próximos da pior condição de saúde.

Esses dados podem ser traduzidos na realização de menos atividade do que o pretendido, diminuição na quantidade de tempo que se dedicava ao trabalho ou a 
outras atividades e estar limitado ou ter dificuldade para realizar alguma atividade regular, como consequência da má saúde física. Este fato chama a atenção para necessidade de implantação de programas de reabilitação, para favorecer o retorno mais rápido e seguro às atividades laborais e físicas.

O estudo apresenta limitações como o número reduzido da amostra e a perda no seguimento da avaliação.

\section{REFERÊNCIAS}

1. Mansur AP, Souza MFM, Timerman A, Avakian SD, Aldrighi JM, Ramires JAF. Tendência do risco de morte por doenças circulatórias, cerebrovasculares e isquêmicas do coração em treze estados do Brasil, de 1980 a 1998. Arq Bras Cardiol. 2006; 87(5):641-8.

2. Klein L, Gheorghiade M. Coronary artery disease and prevention of heart failure. Med Clin North Am. 2004; 88(5):1209-35.

3. Phillips HR, O'Connor CM, Rogers J. Revascularization for heart failure. Am Heart J. 2007; 153(4 Suppl):65-73.

4. Jeremias A, Kaul S, Rosengart TK, Gruberg L, Brown DL. The impact of revascularization on mortality in patients with nonacute coronary artery disease. Am J Med. 2009; 122(2):152-61.

5. Zhang Z, Weintraub WS, Mahoney EM, Spertus JA, Booth J, NUGARA $F$, et al. Relative benefit of coronary artery bypass grafting versus stent-assisted percutaneous coronary intervention for angina pectoris and multivessel coronary disease in women versus men. Am J Cardiol. 2004; 93(4):404-

6. Lavie CJ, Milani RV, Ventura HO, Messerli FH, Murgo JP Cardiac rehabilitation, exercise training and preventive cardiology research. Tex Heart Inst J. 1995; 22(1):44-52.

7. Rozentul LA, Faria WM, Meirelles LR, Brunini TMC. Efeitos da atividade aeróbia sobre a função cardiovascular na fase III da cirurgia de revascularização do miocárdio. Rev Soc Cardiol Estado de São Paulo. 2005; 15(Supl A):9-18.

8. Adams BJ, Carr JG, Ozonoff A, Lauer MS, Balady GJ. Effect of exercise training in supervised cardiac rehabilitation program on prognostic variables from the exercise tolerance test. $A m \mathrm{~J}$ Cardiol. 2008; 101(10):1403-7.

9. Wenger NK. Current status of cardiac rehabilitation. J Am Coll Cardiol. 2008; 51(17):1619-1631.

10. Mozaffarian D, Furberg CD, Psaty BM, Siscovick D. Physical activity and incidence of atrial fibrillation in older adults: the cardiovascular health study. Circulation. 2008; 118(8):800-7.

11. Lee BC, Chen SY, Hsu HC, Su MYM, Wu YW, Chien KL, et al. Effect of cardiac rehabilitation on myocardial perfusion reserve in post-infarction patients. Am J Cardiol, 2008; 101(10):13951402.

12. Dimmeler S, Zeiher AM. Exercise and cardiovascular health get active to "AKTivate" your endothelial nitric oxide synthase. Circulation, 2003; 107(25):3118-20.

13. Williams MA, Ades PA, Hamm LF, Keteyian SJ, Lafontaine TP, Roitman JL, et al. Clinical evidence for a health benefit from cardiac rehabilitation: an update. Am Heart J. 2006; 152(5):83541.

14. Pasquali SK, Alexander KP, Coombs LP, Lytle BL, Peterson ED. Effect of cardiac rehabilitation on functional outcomes after coronary revascularization. Am Heart J. 2003; 145(3):44551.

15. Stahle A, Mattsson E, Ryden L, Unden AL, Nordlander R Improved physical fitness and quality of life following training of elderly patients after acute coronary events. A 1 year followup randomized controlled study. Eur Heart J. 1999; 20(20):147584.

\section{CONCLUSÃO}

$\mathrm{Na}$ amostra estudada, foi observado acréscimo na pontuação dos domínios "capacidade funcional" e "limitação por aspectos físicos" após seis meses da CRVM, o que traduz em aumento da capacidade de realizar atividades que no pré-operatório não eram possíveis. A melhora destes domínios foi mais expressiva em indivíduos que praticaram atividade física regularmente após o procedimento cirúrgico.

16. Ciconelli RM, Ferraz MB, Santos W, Meinão I, Quaresma MR. Tradução para português e validação do questionário genérico de avaliação de qualidade de vida SF 36 (Brasil SF-36). Rev Bras Reumatol. 1999; 39(3):143-50.

17. Weinberg M, Oddone EZ, Sousa GP, Landsman PB. Are health related quality of life measures affected by the mode of administration? J Clin Epidemiol. 1996; 49(2):135-40.

18. Benedetti TRB, Antunes PC, Añez CRR, Mazo GZ, Petroski EL. Reprodutibilidade e validade do Questionário Internacional de Atividade Física (IPAQ) em homens idosos. Rev Bras Med Esporte. 2007; 13(1):11-6.

19. Guedes DP, Lopes CC, Guedes JERP. Reprodutibilidade e validade do Questionário Internacional de Atividade Física em adolescentes. Rev Bras Med Esporte. 2005; 11(2):151-8.

20. Silva MAD, Sousa AGMR, Schargodsky H. Fatores de risco para infarto do miocárdio no Brasil estudo FRICAS. Arq Bras Cardiol. 1998; 71(5):667-75.

21. Swenson JR, O'Connor CM, Barton D, Zyl LTV, Swedberg K, Forman LM, et al. Influence of depression and effect of treatment with sertraline on quality of life after hospitalization for acute coronary syndrome. Am J Cardiol. 2003; 92(11):1271-6.

22. Carvalho T, Cortez AA, Ferraz A, Nóbrega ACL, Brunetto AF Herdy $\mathrm{AH}$, et al. Diretriz de Reabilitação cardiopulmonar $\mathrm{e}$ metabólica: aspectos práticos e responsabilidades. Arq Bras Cardiol. 2006; 86(1):74-82.

23. Stephens MB. Cardiac rehabilitation. Am Fam Physician. 2009; 80(9):955-9.

24. Taylor RS, Brown A, Ebrahim S, Jolliffe J, Noorani H, Rees K, et al. Exercise-based rehabilitation for patients with coronary heart disease: systematic review and meta-analysis of randomized controlled trials. Am J Med. 2004; 116(10):682-92.

25. Takiuti ME, Hueb W, Hiscock SB, Nogueira CRSR, Girardi P, Favarato D, et al. Qualidade de vida após revascularização cirúrgica do miocárdio, angioplastia ou tratamento clínico. Arq Bras Cardiol. 2007; 88(5):537-44.

26. Decker C, Ahmad H, Moreng KL, Maddox TM, Reid KJ, Jones $\mathrm{PG}$, et al. Risk factor management after myocardial infarction: reported adherence and outcomes. Am Heart J. 2009; 157(3):55662.

\section{Correspondência}

Caio Cesar Araújo Morais

Rua Quarenta e Oito, n 76, Sala 01, Espinheiro.

CEP: $52020-060$

Recife - Pernambuco - Brasil.

E-mail: caiocesarfisio@hotmail.com 